\title{
Comparison of Thermal Ablation and Surgery for Low-Risk Papillary Thyroid Microcarcinoma: A Systematic Review and Meta-Analysis
}

\author{
Hyun Jin $\mathrm{Kim}^{1 *}$, Se Jin Cho ${ }^{2 *}$, Jung Hwan Baek ${ }^{3}$ \\ ${ }^{1}$ Department of Radiology, Daejin Medical Center, Bundang Jesaeng General Hospital, Seongnam, Korea; ${ }^{2}$ Department of Radiology, Seoul National \\ University Bundang Hospital, Seoul National University College of Medicine, Seongnam, Korea; ${ }^{3}$ Department of Radiology and Research Institute \\ of Radiology, University of Ulsan College of Medicine, Asan Medical Center, Seoul, Korea
}

Objective: Although thermal ablation is effective in treating low-risk papillary thyroid microcarcinomas (PTMCs), comparison of treatment outcomes between thermal ablation and surgery has not yet been systematically evaluated. This study aimed to compare the efficacy and safety of thermal ablation and surgery for the treatment of low-risk PTMCs.

Materials and Methods: Ovid-MEDLINE and EMBASE databases were searched for studies reporting comparisons of treatment results between thermal ablation and surgery for patients with low-risk PTMC published up to April 6, 2020. The analysis evaluated the efficacy (local tumor recurrence, occurrence of new tumor, metastasis, and rescue surgery) and safety (complication rate) of thermal ablation and surgery.

Results: This systematic review included four studies with a total of 339 PTMCs in 339 patients who underwent thermal ablation and 320 PTMCs in 314 patients who underwent surgery. There was no local tumor recurrence or distant metastasis in either group. There was no significant difference in the pooled proportion of lymph node metastasis $(2.6 \%$ with thermal ablation vs. $3.3 \%$ with surgery, $p=0.65)$, occurrence of new tumors $(1.4 \%$ with thermal ablation vs. $1.3 \%$ with surgery, $p=$ $0.85)$, or rescue surgery $(2.6 \%$ with thermal ablation vs. $1.6 \%$ with surgery, $p=0.62)$. However, the pooled complication rate was significantly higher in the surgery group than in the ablation group $(3.3 \%$ with thermal ablation vs. $7.8 \%$ with surgery, $p=0.03$ ).

Conclusion: Both thermal ablation and surgery are effective and safe options for the management of low-risk PTMCs, with thermal ablation achieving a lower complication rate. Therefore, thermal ablation may be considered as an alternative treatment option for low-risk PTMC in patients who refuse surgery and active surveillance or are ineligible for surgery.

Keywords: Thyroid gland; Papillary thyroid microcarcinoma; Radiofrequency ablation; Thermal ablation; Meta-analysis

\section{INTRODUCTION}

The incidence of papillary thyroid carcinoma (PTC) has

Received: November 2, 2020 Revised: March 3, 2021

Accepted: March 17, 2021

*These authors contributed equally to this work.

Corresponding author: Jung Hwan Baek, MD, PhD, Department of Radiology and Research Institute of Radiology, University of Ulsan College of Medicine, Asan Medical Center, 88 Olympic-ro 43-gil, Songpa-gu, Seoul 05505, Korea.

- E-mail: radbaek@naver.com

This is an Open Access article distributed under the terms of the Creative Commons Attribution Non-Commercial License (https://creativecommons.org/licenses/by-nc/4.0) which permits unrestricted non-commercial use, distribution, and reproduction in any medium, provided the original work is properly cited. recently increased markedly. This is primarily due to the incidental detection of small PTCs and/or early ultrasound (US) screening $[1,2]$. Papillary thyroid microcarcinoma (PTMC), defined as a PTC $\leq 1.0 \mathrm{~cm}$ in size, has a very favorable prognosis, with a disease-specific mortality rate of $<1 \%$ [3]. Despite the indolent nature of PTMC, surgery has been the mainstream treatment [4]. However, this has several drawbacks, such as risks of vocal cord paralysis, hypothyroidism, and hypoparathyroidism. Therefore, the 2015 American Thyroid Association (ATA) guidelines recommended active surveillance (AS) as an alternative first-line option for the management of low-risk PTMCs because it reduces unnecessary surgery while showing favorable outcomes $[4,5]$. However, there is a risk of tumor 
size enlargement, local tumor recurrence, and distant and lymph node (LN) metastasis, and there are ongoing discussions to date about patient anxiety and patient resistance to AS $[6,7]$.

Accumulating evidence suggests that thermal ablation techniques, including radiofrequency ablation (RFA), microwave ablation (MWA), and laser ablation (LA), are effective and safe treatment modalities for benign thyroid nodules [8-11] and recurrent thyroid cancers [12-15]. Several recent clinical trials have evaluated the thermal ablation of low-risk PTMCs [11,16-19]. Although these studies reported that thermal ablation is an effective and safe option for treating low-risk PTMCs, thermal ablation is not recommended for primary thyroid cancer according to the ATA guidelines [4]. Thermal ablation has not been established as a treatment tool for low-risk PTMCs; however, the 2017 Korean RFA guidelines and Italian opinion statement presented the potential therapeutic role of RFA in primary thyroid cancer in selected patients who refuse or are ineligible for surgery $[20,21]$. Conversely, few studies have compared the efficacy and safety of surgery and thermal ablation [22-26]. Therefore, in this study, we aimed to evaluate and compare the efficacy and safety of thermal ablation and surgery for the treatment of low-risk PTMCs by performing a systematic review and meta-analysis.

\section{MATERIALS AND METHODS}

This systematic review and meta-analysis was performed according to the Preferred Reporting Items for Systematic Reviews and Meta-analyses (PRISMA) guidelines [27]. The need for informed consent was waived due to the study being a systematic review and meta-analysis.

\section{Literature Search}

A search of MEDLINE and EMBASE databases was performed to find original literature reporting a comparison of treatment results between thermal ablation and surgery in patients with low-risk PTMCs. The following search terms were used: ((surgery) OR (surgical) OR (operation) OR (ablation)) AND ((papillary thyroid microcarcinoma) OR (thyroid micropapillary carcinoma) OR (papillary thyroid micro-carcinoma)) AND ((comparative) OR (comparison) OR (versus)). No beginning search date was set, with the search for literature published until April 6, 2020. The search was limited to English-language publications. The bibliographies of the relevant articles were searched to identify any other appropriate articles.

\section{Inclusion Criteria}

Studies satisfying the following criteria were included: 1) studies on patients with low-risk PTMCs and 2) studies comparing treatment results between thermal ablation and surgery.

\section{Exclusion Criteria}

Studies or subsets of studies were excluded if any of the following criteria were met: 1) case reports or case series including < 20 patients; 2) letters, editorials, conference abstracts, consensus statements, guidelines, and review articles; 3 ) articles not focusing on the specified topic; 4) articles with, or with suspicion of, overlapping populations; 5 ) articles not containing treatment results for both thermal ablation and surgery; and 6) articles without reference standards based on histopathological tests.

Two radiologists, with 6 and 7 years of experience in thyroid imaging, respectively, independently performed the literature search and selection.

\section{Data Extraction}

The following data were extracted using standardized forms according to the PRISMA guidelines [27]: 1) characteristics of the article, including main author, year of publication, duration of patient recruitment, institution, country of origin, study design (prospective or retrospective), enrollment criteria (summary of inclusion and exclusion) for treatment, comparison arm (surgery vs. specific form of thermal ablation), number of patients, mean patient age, and male to female ratio according to the treatment methods; 2 ) details of the surgery and thermal ablation including the maximum diameter and mean volume of the index tumor before treatment, surgical method (lobectomy only, lobectomy with central neck dissection [CND], total thyroidectomy only, and total thyroidectomy with CND), the methods of ablation (MWA, LA, and RFA), and devices used, needle tip, and mean power output; 3) results of ablation and details of follow-up, including mean follow-up duration, rates of local tumor recurrence (recurrence of treated tumor), rates of appearance of new cancer separate from the index tumor (detection of new tumor other than the treated one), rates of $L N$ metastasis, rates of distant metastasis, rates of patients who underwent delayed surgery during follow-up after initial treatment, number of treatment sessions, volume 
reduction rates $\{$ VRRs; VRR during follow-up was calculated as ([initial nodular volume - final nodular volume] x 100)/ initial nodular volume\}, and rates of tumor disappearance in patients undergoing thermal ablation; and 4) details of the complications associated with treatment were reclassified by the authors using the reporting standards of the Society of Interventional Radiology [28]. Major complications were defined as adverse events associated with substantial morbidity or disability, increased level of care, hospital admission, or substantial prolongation of hospital stay. Other complications were considered minor, while unintended consequences that did not require therapy or medical treatment, as well as undesired consequences of the procedure, were defined as side effects, according to previous studies $[29,30]$. Voice change and hypoparathyroidism were considered major complications if they had a duration of $>1$ month, but were considered minor complications if they had a duration of $<1$ month. If the duration was not available, they were considered major complications. However, occurrences immediately following ablation, including transient voice change, minimal or asymptomatic hematoma, bleeding, parenchymal edema, and tolerable mild pain or discomfort not requiring medication, were regarded as neither complications nor side effects.

\section{Quality Assessment}

Two authors independently performed data extraction and quality assessment using the risk of bias for nonrandomized studies (RoBANS) tool for non-randomized controlled trials [31].

\section{Data Synthesis and Analyses}

The primary outcomes of the current systematic review and meta-analysis were the pooled proportions of local tumor recurrence, metastasis, and complications. Pooled proportions were calculated using an inverse-variance weighting model [32]. A random-effects meta-analysis of proportions was used to calculate the overall proportions. Study heterogeneity was evaluated using the Higgins inconsistency index (I2), with substantial heterogeneity indicated by an I2 value $>50 \%$ [33]. In addition, metaregression analysis was performed to compare the proportion of local tumor recurrence, metastasis, and complications between the two treatment methods (surgery vs. ablation). All statistical analyses were conducted by one author (with 2 years of experience in conducting systematic reviews and meta-analysis), using the "meta" package in $\mathrm{R}$ version 3.6.1 (http://www.r-project.org/).

\section{RESULTS}

\section{Literature Search}

The article selection process is illustrated in Figure 1. The initial systematic literature search identified 334 articles. After duplicates were removed, 255 articles were screened for eligibility. Of these, 250 were excluded after the abstracts were reviewed. The full texts of the remaining five articles were reviewed, and one article was excluded because of a partially overlapping patient cohort [26]. Finally, four eligible articles were included in the analysis [22-25].

\section{Characteristics of the Included Studies}

Table 1 shows the detailed characteristics of the four studies and the types of ablation used. A total of 314 patients were included in the surgery group and 339 patients in the ablation group. All four studies had a retrospective design. The criteria for treatment were generally similar across the studies, including low-risk PTMC diagnosed by cytology or biopsy, absence of $L N$ or distant metastasis, and absence of visible gross extrathyroidal extension on US. Three of the studies enrolled patients with solitary PTMC $[22,24,25]$, while one study did not present information about the number of tumors in enrolled patients [23]. Surgery or ablation was selected according to the patient's general condition, wishes, or other reasons [22-25]. As regards the ablation method, two studies used MWA [22,23], one study used LA [25], and one study used RFA [24]. The eligible studies included patient numbers ranging from 45 to 143 in the surgery group and from 36 to 168 in the ablation group. All studies included patients with single PTMCs by US; however, in one study, several patients were revealed to have multiple tumors after surgery [24]. The mean ages of the included patients ranged from 41.6 to 49.2 years in the surgery group and from 41.5 to 47.4 years in the ablation group. All studies included more female than male [22-25].

\section{Characteristics of the Surgery and Ablation Methods}

The detailed characteristics of the surgery and ablation methods are presented in Table 2. In three studies, all of the patients subjected to surgery underwent lobectomy with CND $[22,23,25]$. In one study, 19 of the 80 patients 


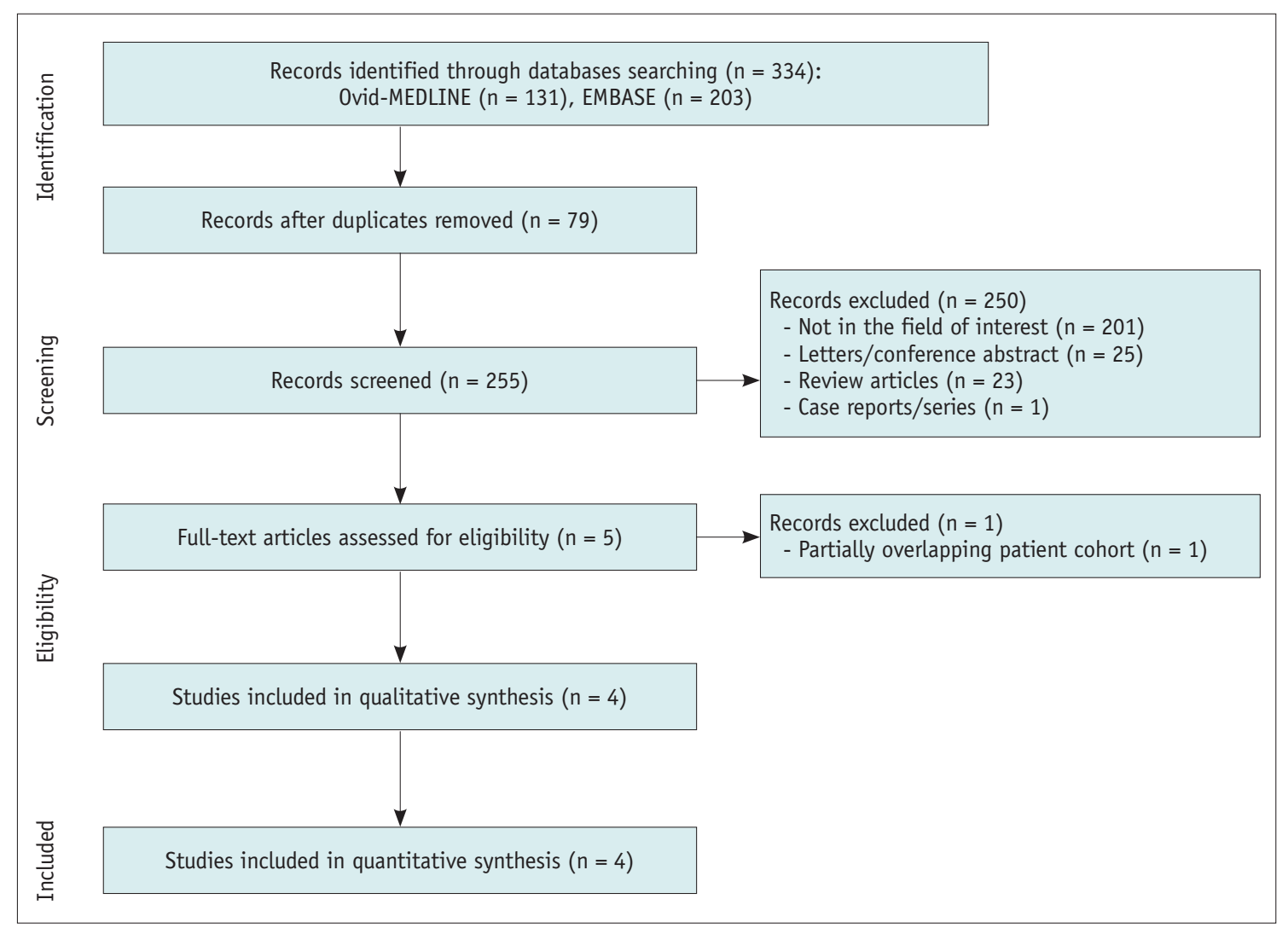

Fig. 1. Flow diagram of the study selection process.

subjected to surgery underwent lobectomy without CND, 39 underwent lobectomy with CND, 8 underwent total thyroidectomy without CND, and 14 underwent total thyroidectomy with CND [24]. All patients treated with ablation underwent only one treatment session [22-25]. The maximal diameter of the index tumor ranged from 5 to $8.1 \mathrm{~mm}$ in the surgery group and from 4.7 to $8.9 \mathrm{~mm}$ in the ablation group [23-25]. The mean volume ranged from 41.6 to $132.7 \mathrm{~mm}^{3}$ in the surgery group $[24,25]$ and from 43.2 to $175.9 \mathrm{~mm}^{3}$ in the ablation group [22,24,25].

\section{Comparison of the Results of Surgery and Ablation}

The results of surgery and ablation are presented in Table 3. Except for one study [23], the mean follow-up duration after treatment was over 2 years in both surgery and ablation groups, ranging from 27.5 to 63.6 months in the surgery group and from 25.1 to 64.2 months in the ablation group. The mean follow-up period was 35.8 months for the surgery group and 35.6 months for the ablation group. In three studies $[22,24,25]$, no local tumor recurrence or distant metastasis was observed during follow-up after surgery or ablation. A new tumor separate from the treated primary tumor developed in $1.3 \%$ and $1.4 \%$ of patients in the surgery and ablation groups, respectively. LN metastasis developed in $3.3 \%$ and $2.6 \%$ of the surgery and ablation groups, respectively. In the surgery group, $1.6 \%$ underwent rescue surgery: two patients with a new tumor [22,25] and one patient with LN metastasis [22]. In the ablation group, $2.6 \%$ underwent delayed surgery: three patients with LN metastasis [22,25] and one patient with a new tumor in the contralateral lobe [25]. There was no significant difference between surgery and ablation in the proportion of patients with local tumor recurrence $(p=0.98)$, new tumor separate from the primary tumor $(p=0.85)$, LN metastasis $(p=0.65)$, distant metastasis $(p=0.90)$, or rescue surgery $(p=0.62)$. Only the pooled proportion of distant metastasis after thermal ablation showed substantial heterogeneity $\left(\mathrm{I}^{2}=58.8 \%\right)$.

\section{Comparison of Complications Following Surgery and Ablation}

The complications following surgery and ablation are presented in Tables 4 and 5, respectively. Complications occurred in $7.8 \%$ and $3.3 \%$ of patients who underwent surgery and ablation, respectively. All of the complications that occurred were major complications, and the frequency 


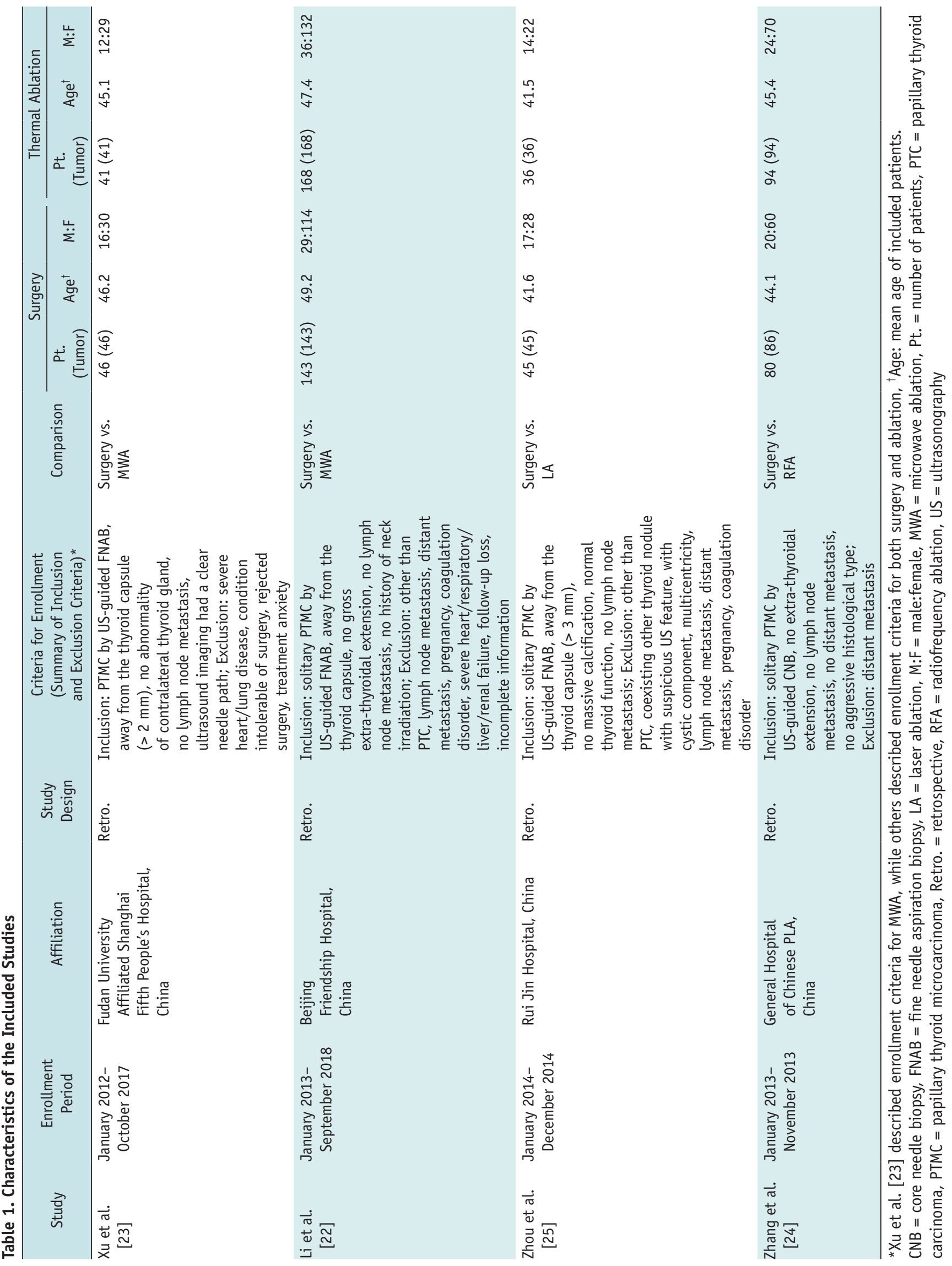



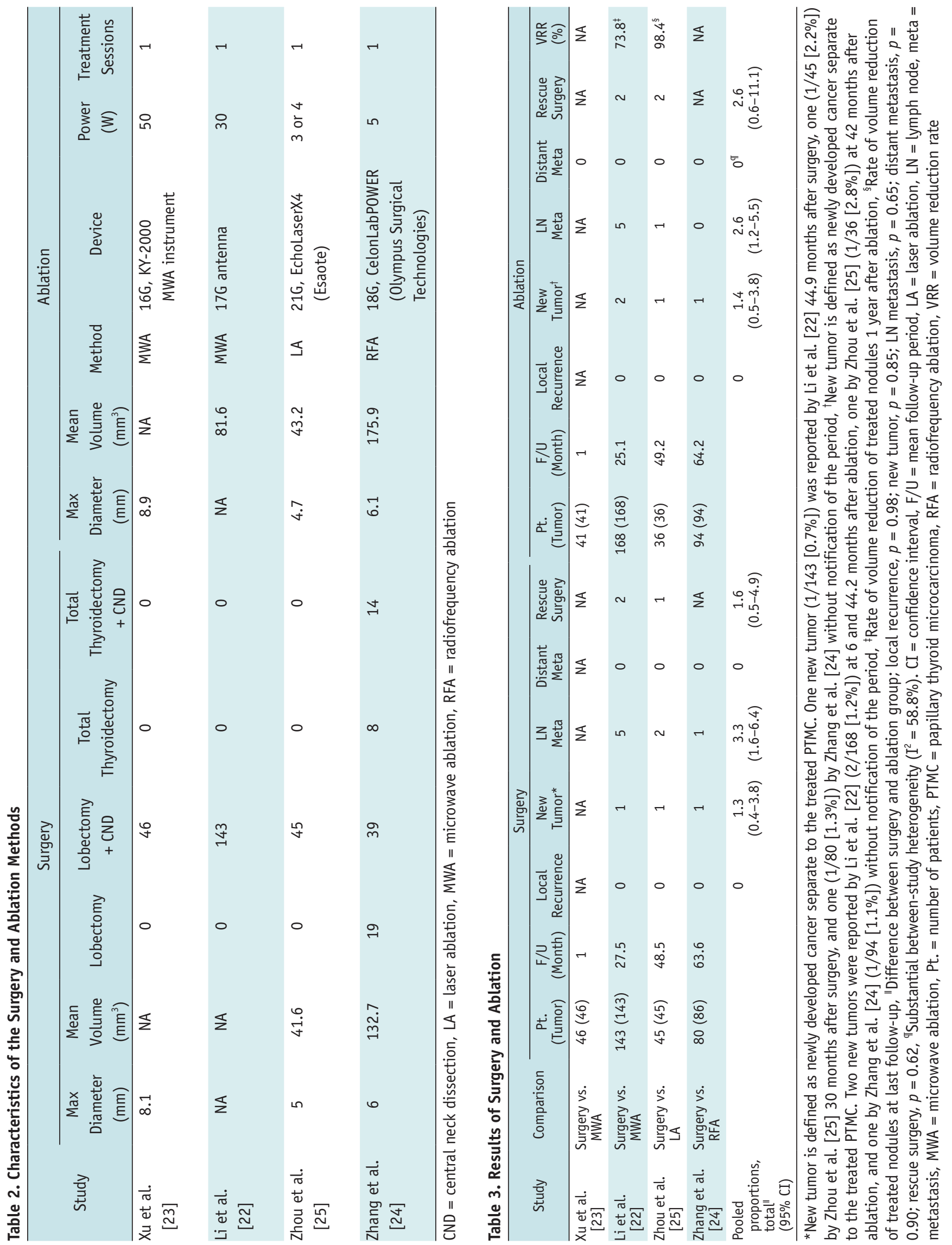


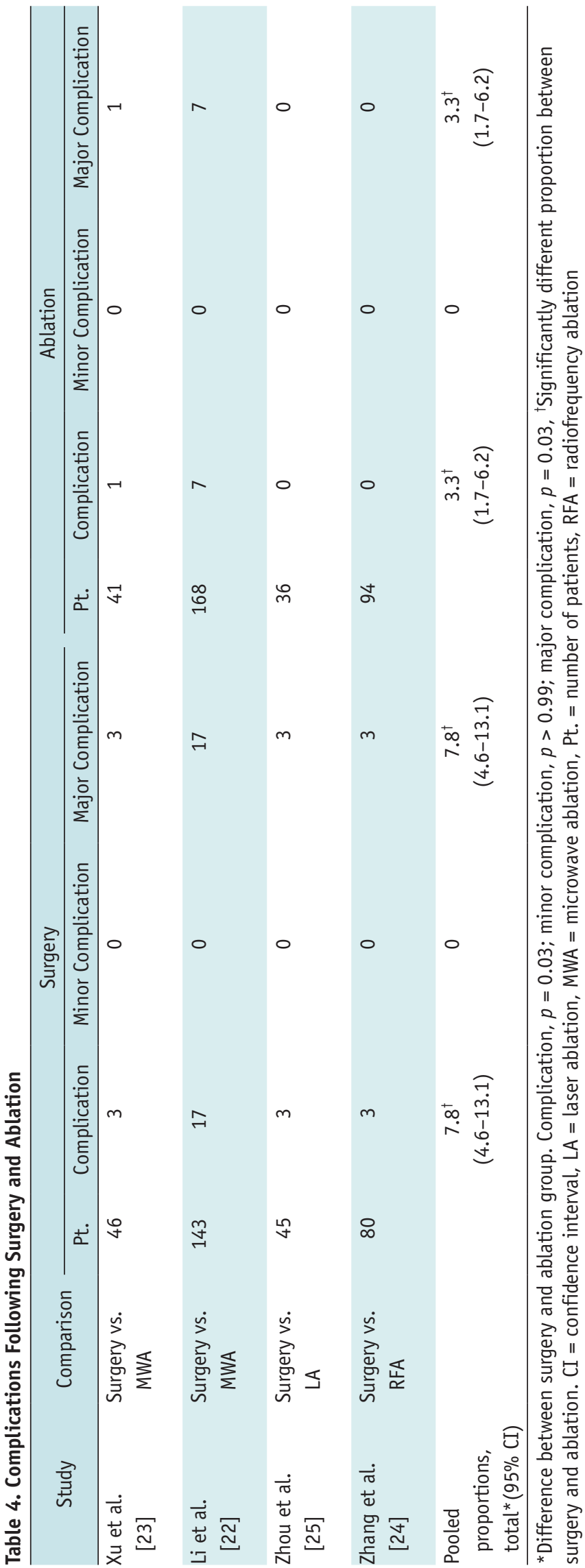

of overall and major complications was significantly higher in the surgery group than in the ablation group $(p=0.03)$. Complications included hoarseness in $4.3 \%$ of patients in the surgery group and $3.3 \%$ of patients in the ablation group, and hypoparathyroidism and wound infection in $3.2 \%$ and $1 \%$ of patients in the surgery group, respectively, with neither of the latter two complications occurring in the ablation group. Among the patients who developed hoarseness, the symptom was permanent in three patients in the surgery group $(1.5 \%)[22,24]$ and in one patient in the ablation group $(0.8 \%)$ [22]. Figure 2 shows the forest plots of pooled proportions of new tumors separate from the treated primary tumor, LN metastasis, and complications after surgery and thermal ablation, respectively.

\section{Quality Assessment of the Studies}

The quality of the included studies was assessed according to the RoBANS criteria, and the results are presented in Figure 3. All studies showed a low risk of bias in selective reporting, outcome evaluation, measurement of exposure, confounding variables, comparability of participants, and selection of patient group domains. However, all of the studies showed an unclear risk of bias in the blinding of the outcome assessment domain, as they did not make clear statements about patient/investigator blinding [22-25]. One study showed an unclear risk of incomplete outcome data because not all of the enrolled patients were followed up [22], whereas the other three studies showed a low risk of incomplete outcome data [23-25].

\section{DISCUSSION}

The current systematic review and meta-analysis compared the efficacy and safety of surgery and thermal ablation for the treatment of low-risk PTMCs in four studies, including a total of 320 PTMCs in 314 patients in the surgery group and 339 PTMCs in 339 patients in the thermal ablation group. There was no local tumor recurrence or distant metastasis in either group. The pooled proportions of $L N$ metastasis $(p=$ $0.65)$ and patients who underwent rescue surgery $(p=0.62)$ were not significantly different between the two groups. However, the pooled complication rate was significantly higher in the surgery group than in the ablation group $(7.8 \%$ vs. $3.3 \%, p=0.03)$. Therefore, this meta-analysis demonstrated that thermal ablation is a safe treatment modality for low-risk PTMCs, with a similar efficacy as surgery. 
Table 5. Comparison of Major Complications Following Surgery and Ablation

\begin{tabular}{|c|c|c|c|c|}
\hline \multirow{2}{*}{ Study } & \multicolumn{3}{|c|}{ Surgery } & \multirow{2}{*}{$\begin{array}{c}\text { Ablation } \\
\text { Hoarseness }\end{array}$} \\
\hline & Hoarseness & Hypoparathyroidism & Wound Infection & \\
\hline Xu et al. [23] & 2 & 0 & 1 & 1 \\
\hline Li et al. [22] & 7 & 10 & 0 & 7 \\
\hline Zhou et al. [25] & 2 & 1 & 0 & 0 \\
\hline Zhang et al. [24] & 2 & 1 & 0 & 0 \\
\hline Pooled proportions, total (95\% CI) & $4.3(2.5-7.2)$ & $3.2(1.1-8.9)$ & $1.0(0.3-3.5)$ & $3.3(1.7-6.2)$ \\
\hline
\end{tabular}

$\mathrm{CI}=$ confidence interval

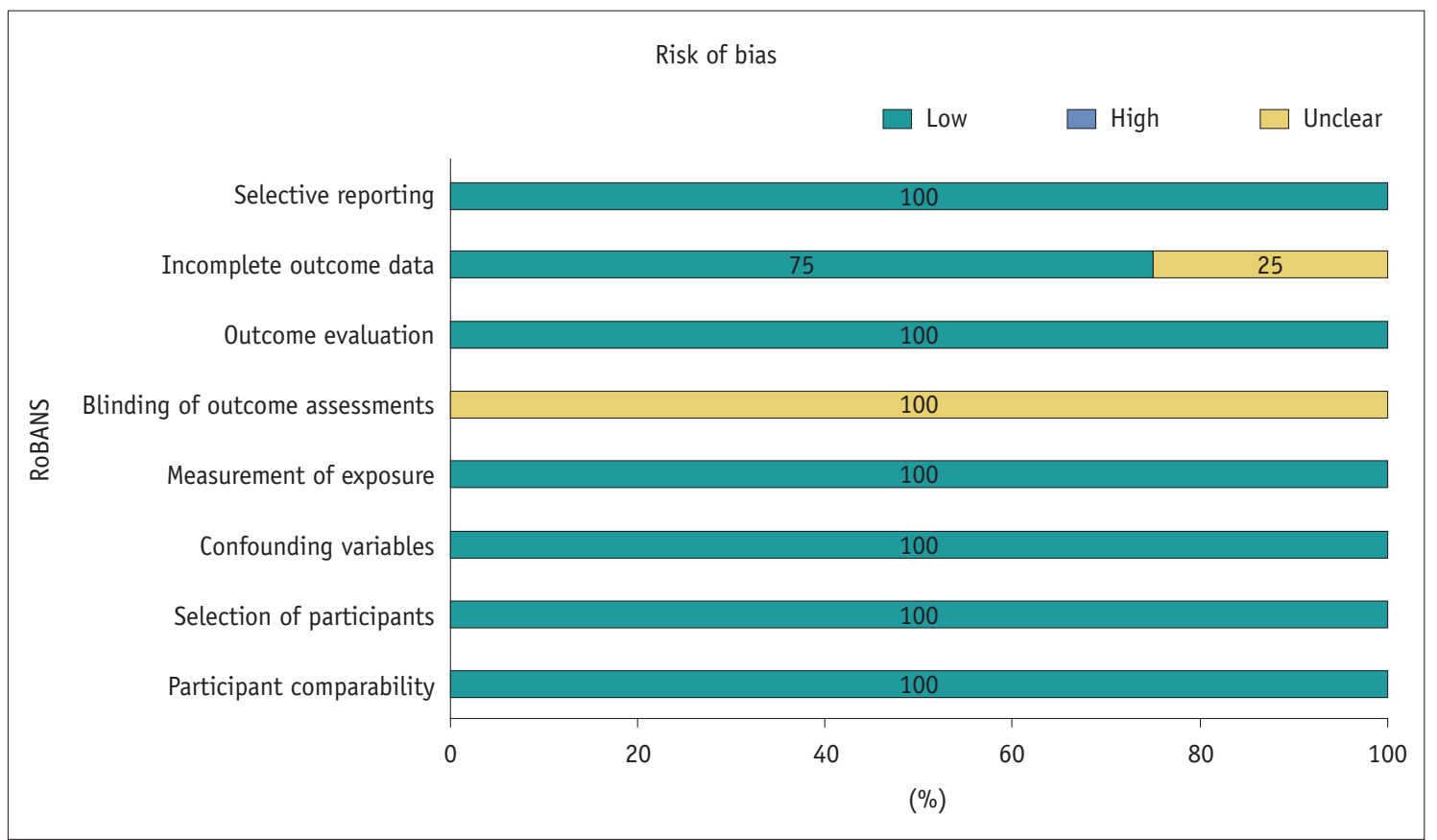

Fig. 2. The quality assessment of the included studies according to the RoBANS criteria. RoBANS = risk of bias for nonrandomized studies

The definition of low-risk PTMC has few discrepancies among guidelines. The recent Japanese guidelines for AS suggested that clinical T1aNOMO PTMCs without indication of immediate surgery are low-risk PTMCs and candidates for AS [34]. Indications for immediate surgery include the presence of LN metastasis, aggressive subtype of PTC on cytology, apparent invasion into the recurrent laryngeal nerve or trachea, tumors adherent to the trachea or located near the course of the recurrent laryngeal nerve, patients associated with other thyroid or parathyroid disease requiring surgery, and age $<20$ years.

The standard treatment for PTMC is surgery, usually unilateral lobectomy [4]. However, because of the indolent nature of this tumor, the 2015 ATA guidelines recommended AS as an alternative first-line option for the management of low-risk PTMCs [4]. A recent systematic review and metaanalysis found that AS showed a low proportion of tumor size enlargement and LN metastasis over a 5-year follow-up (pooled proportions of $5.3 \%$ and $1.6 \%$, respectively) [35]. However, a large proportion (ranging from $8.7 \%$ to $32 \%$ ) of patients underwent delayed or rescue surgery, with many reasons for surgery (range of proportions, 50\% to 69\%) being those other than size enlargement or LN metastasis, that is, a considerable proportion of the surgeries performed were not rescue surgery. The authors presumed that anxiety was one of the main reasons for delayed surgery [35]. Although AS has gradually become more accepted in clinical practice, patient anxiety remains an unresolved issue $[6,7]$.

Regarding thermal ablation for PTMC, only $1.1 \%$ of patients in a recent systematic review and $2.6 \%$ of patients in our meta-analysis underwent rescue surgery after thermal ablation, with none of the patients undergoing delayed surgery because of anxiety about tumor progression. A previous systematic review showed that no patient 


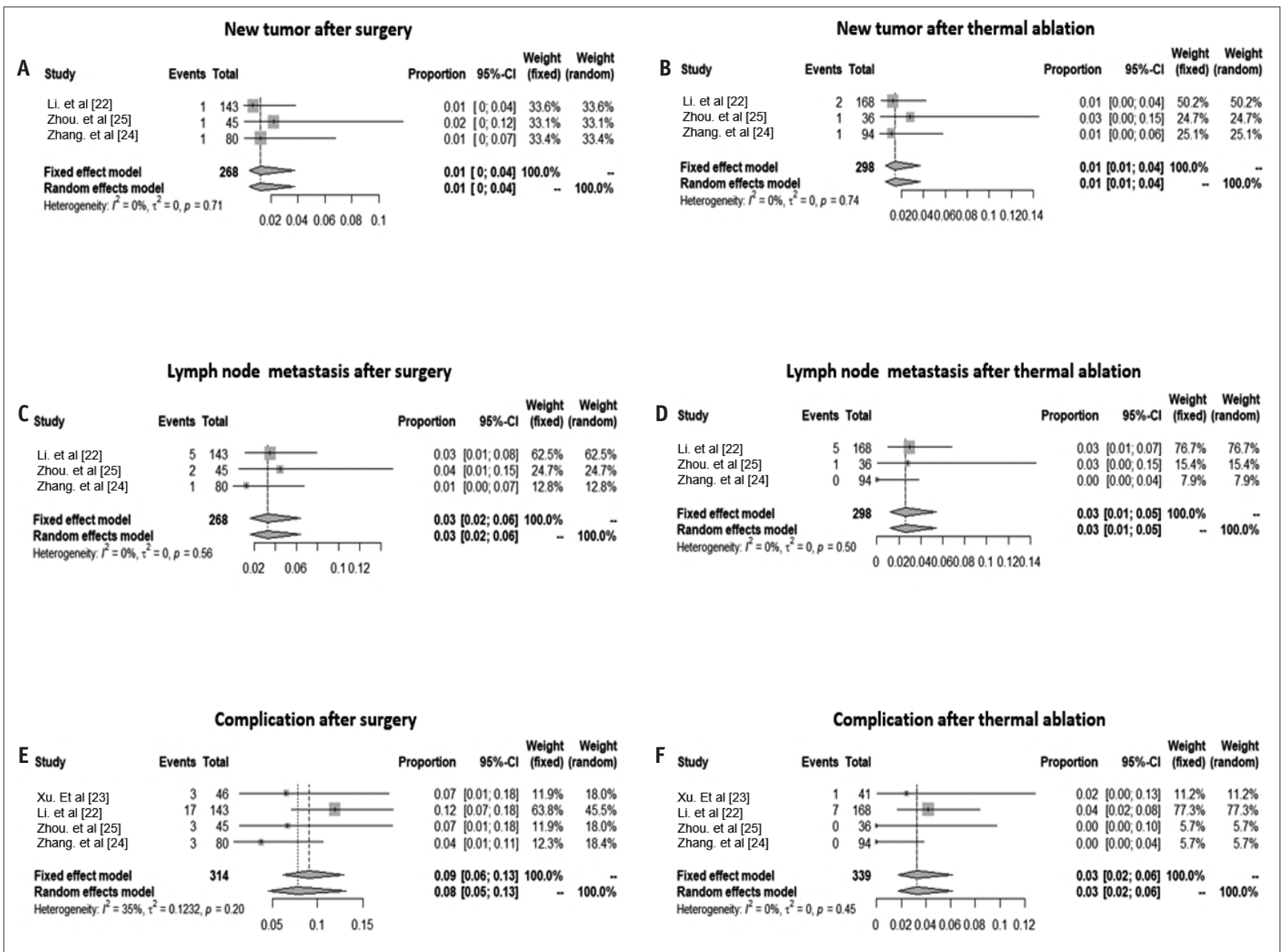

Fig. 3. Forest plots of pooled proportions of $(A)$ new tumor separate from treated primary tumor after surgery, (B) new tumor separate from treated primary tumor after thermal ablation, (C) lymph node metastasis after surgery, (D) lymph node metastasis after thermal ablation, (E) complication after surgery, and (F) complication after thermal ablation. $\mathrm{CI}=$ confidence interval

experienced local tumor recurrence or distant metastasis during follow-up, while $0.4 \%$ of patients experienced $L N$ metastasis and $0.2 \%$ of patients developed a new PTMC, which was successfully treated by additional ablation [36]. No life-threatening complications were reported after thermal ablation in the included studies. Moreover, none of the patients developed persistent hypothyroidism requiring lifelong thyroid hormone replacement. With regard to quality of life, thermal ablation may have an advantage over surgery in that it has a lower risk of persistent hypothyroidism and there is no scarring [37-39]. It is worth noting that patients who had undergone thermal ablation in these studies included not only those who were ineligible for surgery, but also those who refused it. Considering the high incidence of delayed or rescue surgery during AS and acceptable treatment results of thermal ablation, thermal ablation may be considered as an alternative to AS or immediate surgery for patients with low-risk PTMCs. However, thermal ablation is a surgeon-dependent technique and involves a potential risk of hidden malignant tumors and hidden LN metastasis. Therefore, as with AS, thermal ablation of PTMC requires strict inclusion criteria and a detailed pretreatment imaging work-up, including contrast-enhanced CT $[19,40]$. This should be performed by an expert surgeon. In addition, as thermal ablation for primary PTMC was recently introduced, the follow-up periods in the analyzed studies were shorter than those of surgery, and randomized controlled studies have yet to be performed. However, Cho et al. [41] recently reported more than 5-year follow-up results of RFA for low-risk PTMCs in 84 tumors. The study showed favorable outcomes, including complete disappearance in $98.8 \%$ of tumors with $1.4 \%$ of major complications during the 72 months of the mean follow-up period. There was no local tumor progression, $\mathrm{LN}$, 
or distant metastasis. This result is not different from that of previous short-term follow-up studies. More long-term follow-up studies and randomized controlled studies are necessary to bridge the evidence gaps.

In our study, almost all patients in the surgery group (306 of 314 patients) underwent prophylactic CND rather than thyroidectomy alone. According to the 2015 ATA guidelines, thyroidectomy without prophylactic CND is appropriate for patients with small (T1 or T2 stage), noninvasive tumors and clinically uninvolved central neck LNs. Moreover, a recent meta-analysis demonstrated that patients who underwent prophylactic CND had significantly higher incidence rates of several complications (transient recurrent laryngeal nerve injury, transient hypocalcemia, and permanent hypocalcemia) [42]. Therefore, the pooled proportion of complications in the surgery group might be higher than that in populations who did not undergo prophylactic CND. Thus, there is a limitation in the direct comparison between surgery and thermal ablation.

This study had a few limitations. First, the number of included studies was relatively small, and three methods of thermal ablation were mixed in this study because thermal ablation for the treatment of PTMC was recently introduced. In addition, few studies have compared thermal ablation with surgery. Second, although the follow-up period in three of the four included studies was over 2 years, it was not long enough to conclusively evaluate the treatment outcome. Third, the criteria for ablation and surgery were not identical across the included studies, although all of the studies included patients with low-risk PTMCs. In addition, there were ambiguities in the criteria for selection of the treatment method in the included studies. In all of the analyzed studies, inoperable patients were treated with thermal ablation, but the reasons for refusing surgery are not clear. Finally, the included studies were concentrated regionally in China. Further well-designed prospective studies with large populations and long-term follow-up are needed to clarify the efficacy of thermal ablation in low-risk PTMCs.

In conclusion, this systematic review and meta-analysis showed that both thermal ablation and surgery are effective and safe options for the management of low-risk PTMCs, with thermal ablation achieving a lower complication rate. Therefore, in future guidelines, thermal ablation may be considered as an alternative treatment option for lowrisk PTMCs in patients who refuse surgery and AS or are ineligible for surgery.
Conflicts of Interest

The authors have no potential conflicts of interest to disclose.

\section{Author Contributions}

Conceptualization: all authors. Data curation: Hyun Jin Kim, Se Jin Cho. Formal analysis: Hyun Jin Kim, Se Jin Cho. Investigation: Hyun Jin Kim, Se Jin Cho. Methodology: all authors. Writing — original draft: Hyun Jin Kim, Se Jin Cho. Writing - review \& editing: all authors.

\author{
ORCID iDs \\ Hyun Jin Kim \\ https://orcid.org/0000-0002-3411-3690 \\ Se Jin Cho \\ https://orcid.org/0000-0001-6450-7554 \\ Jung Hwan Baek \\ https://orcid.org/0000-0003-0480-4754
}

\section{REFERENCES}

1. Ahn HS, Kim HJ, Welch HG. Korea's thyroid-cancer "epidemic"-screening and overdiagnosis. N Engl J Med 2014;371:17651767

2. Vaccarella S, Franceschi S, Bray F, Wild CP, Plummer M, Dal Maso L. Worldwide thyroid-cancer epidemic? The increasing impact of overdiagnosis. N Engl J Med 2016;375:614-617

3. Hay ID. Management of patients with low-risk papillary thyroid carcinoma. Endocr Pract 2007;13:521-533

4. Haugen BR, Alexander EK, Bible KC, Doherty GM, Mandel SJ, Nikiforov YE, et al. 2015 American Thyroid Association management guidelines for adult patients with thyroid nodules and differentiated thyroid cancer: the American Thyroid Association guidelines task force on thyroid nodules and differentiated thyroid cancer. Thyroid 2016;26:1-133

5. Ito $Y$, Miyauchi $A$, Inoue $H$, Fukushima M, Kihara $M$, Higashiyama T, et al. An observational trial for papillary thyroid microcarcinoma in Japanese patients. World J Surg 2010;34:28-35

6. Davies L, Roman BR, Fukushima M, Ito Y, Miyauchi A. Patient experience of thyroid cancer active surveillance in Japan. JAMA Otolaryngol Head Neck Surg 2019;145:363-370

7. Brito JP, Moon JH, Zeuren R, Kong SH, Kim YG, Iñiguez-Ariza NM, et al. Thyroid cancer treatment choice: a pilot study of a tool to facilitate conversations with patients with papillary microcarcinomas considering treatment options. Thyroid 2018;28:1325-1331

8. Mauri G, Nicosia L, Della Vigna P, Varano GM, Maiettini D, Bonomo G, et al. Percutaneous laser ablation for benign and malignant thyroid diseases. Ultrasonography 2019;38:25-36

9. Sim JS, Baek JH. Long-term outcomes following thermal 
ablation of benign thyroid nodules as an alternative to surgery: the importance of controlling regrowth. Endocrinol Metab (Seoul) 2019;34:117-123

10. Huh JY, Baek JH, Choi H, Kim JK, Lee JH. Symptomatic benign thyroid nodules: efficacy of additional radiofrequency ablation treatment session--prospective randomized study. Radiology 2012;263:909-916

11. Yue W, Wang S, Yu S, Wang B. Ultrasound-guided percutaneous microwave ablation of solitary T1NOMO papillary thyroid microcarcinoma: initial experience. Int $\mathrm{J}$ Hyperthermia 2014;30:150-157

12. Choi Y, Jung SL, Bae JS, Lee SH, Jung CK, Jang J, et al. Comparison of efficacy and complications between radiofrequency ablation and repeat surgery in the treatment of locally recurrent thyroid cancers: a single-center propensity score matching study. Int J Hyperthermia 2019;36:359-367

13. Chung SR, Baek JH, Choi YJ, Lee JH. Longer-term outcomes of radiofrequency ablation for locally recurrent papillary thyroid cancer. Eur Radiol 2019;29:4897-4903

14. Kim JH, Yoo WS, Park YJ, Park DJ, Yun TJ, Choi SH, et al. Efficacy and safety of radiofrequency ablation for treatment of locally recurrent thyroid cancers smaller than $2 \mathrm{~cm}$. Radiology 2015;276:909-918

15. Lim HK, Baek JH, Lee JH, Kim WB, Kim TY, Shong YK, et al. Efficacy and safety of radiofrequency ablation for treating locoregional recurrence from papillary thyroid cancer. Eur Radiol 2015;25:163-170

16. Jeong SY, Baek JH, Choi YJ, Chung SR, Sung TY, Kim WG, et al. Radiofrequency ablation of primary thyroid carcinoma: efficacy according to the types of thyroid carcinoma. Int J Hyperthermia 2018;34:611-616

17. Ji L, Wu Q, Gu J, Deng X, Zhou W, Fan X, et al. Ultrasoundguided percutaneous laser ablation for papillary thyroid microcarcinoma: a retrospective analysis of 37 patients. Cancer Imaging 2019;19:16

18. Teng DK, Li HQ, Sui GQ, Lin YQ, Luo Q, Fu P, et al. Preliminary report of microwave ablation for the primary papillary thyroid microcarcinoma: a large-cohort of 185 patients feasibility study. Endocrine 2019;64:109-117

19. Lim HK, Cho SJ, Baek JH, Lee KD, Son CW, Son JM, et al. USguided radiofrequency ablation for low-risk papillary thyroid microcarcinoma: efficacy and safety in a large population. Korean J Radiol 2019;20:1653-1661

20. Garberoglio R, Aliberti C, Appetecchia M, Attard M, Boccuzzi G, Boraso $F$, et al. Radiofrequency ablation for thyroid nodules: which indications? The first Italian opinion statement. $J$ Ultrasound 2015;18:423-430

21. Kim JH, Baek JH, Lim HK, Ahn HS, Baek SM, Choi YJ, et al. 2017 thyroid radiofrequency ablation guideline: Korean Society of Thyroid Radiology. Korean J Radiol 2018;19:632655

22. Li J, Liu Y, Liu J, Yang P, Hu X, Qian L. A comparative study of short-term efficacy and safety for thyroid micropapillary carcinoma patients after microwave ablation or surgery. Int $J$
Hyperthermia 2019;36:640-646

23. Xu B, Zhou NM, Cao WT, Gu SY. Comparative study on operative trauma between microwave ablation and surgical treatment for papillary thyroid microcarcinoma. World J Clin Cases 2018;6:936-943

24. Zhang M, Tufano RP, Russell J0, Zhang Y, Zhang Y, Qiao Z, et al. Ultrasound-guided radiofrequency ablation versus surgery for low-risk papillary thyroid microcarcinoma: results of over 5 years' follow-up. Thyroid 2020;30:408-417

25. Zhou W, Ni X, Xu S, Zhang L, Chen Y, Zhan W. Ultrasoundguided laser ablation versus surgery for solitary papillary thyroid microcarcinoma: a retrospective study. Int J Hyperthermia 2019;36:897-904

26. Li J, Liu Y, Liu J, Qian L. Ultrasound-guided percutaneous microwave ablation versus surgery for papillary thyroid microcarcinoma. Int J Hyperthermia 2018;34:653-659

27. Liberati A, Altman DG, Tetzlaff J, Mulrow C, Gøtzsche PC, Ioannidis JP, et al. The PRISMA statement for reporting systematic reviews and meta-analyses of studies that evaluate health care interventions: explanation and elaboration. Ann Intern Med 2009;151:W65-W94

28. Ahmed M, Solbiati L, Brace CL, Breen DJ, Callstrom MR, Charboneau JW, et al. Image-guided tumor ablation: standardization of terminology and reporting criteria--a 10year update. J Vasc Interv Radiol 2014;25:1691-1705.e4

29. Baek JH, Lee JH, Sung JY, Bae JI, Kim KT, Sim J, et al. Complications encountered in the treatment of benign thyroid nodules with US-guided radiofrequency ablation: a multicenter study. Radiology 2012;262:335-342

30. Kim C, Lee JH, Choi YJ, Kim WB, Sung TY, Baek JH. Complications encountered in ultrasonography-guided radiofrequency ablation of benign thyroid nodules and recurrent thyroid cancers. Eur Radiol 2017;27:3128-3137

31. Kim SY, Seo HJ, Lee YJ, Park JE. Study design algorithm for medical literature of intervention (DAMI) and risk of bias for nonrandomized studies (robans) ver 2.0 by HIRA. Seoul: Health Insurance Review \& Assessment Service, 2013

32. Suh CH, Park SH. Successful publication of systematic review and meta-analysis of studies evaluating diagnostic test accuracy. Korean J Radiol 2016;17:5-6

33. Higgins JP, Thompson SG, Deeks JJ, Altman DG. Measuring inconsistency in meta-analyses. BMJ 2003;327:557-560

34. Sugitani I, Ito Y, Takeuchi D, Nakayama H, Masaki C, Shindo $\mathrm{H}$, et al. Indications and strategy for active surveillance of adult low-risk papillary thyroid microcarcinoma: consensus statements from the Japan Association of Endocrine Surgery Task Force on management for papillary thyroid microcarcinoma. Thyroid 2021;31:183-192

35. Cho SJ, Suh CH, Baek JH, Chung SR, Choi YJ, Chung KW, et al. Active surveillance for small papillary thyroid cancer: a systematic review and meta-analysis. Thyroid 2019;29:13991408

36. Cho SJ, Baek JH, Chung SR, Choi YJ, Lee JH. Thermal ablation for small papillary thyroid cancer: a systematic review. Thyroid 
2019;29:1774-1783

37. Ding B, Yu JF, Sun W, Ma NF. Surgical safety analysis of retaining the glands in papillary thyroid microcarcinoma. Eur Rev Med Pharmacol Sci 2017;21:234-238

38. Goldfarb M, Casillas J. Thyroid cancer-specific quality of life and health-related quality of life in young adult thyroid cancer survivors. Thyroid 2016;26:923-932

39. Zhou W, Zhang L, Zhan W, Jiang S, Zhu Y, Xu S. Percutaneous laser ablation for treatment of locally recurrent papillary thyroid carcinoma <15 mm. Clin Radiol 2016;71:1233-1239

40. Suh CH, Baek JH, Choi YJ, Lee JH. Performance of CT in the preoperative diagnosis of cervical lymph node metastasis in patients with papillary thyroid cancer: a systematic review and meta-analysis. AJNR Am J Neuroradiol 2017;38:154-161

41. Cho SJ, Baek SM, Lim HK, Lee KD, Son JM, Baek JH. Longterm follow-up results of ultrasound-guided radiofrequency ablation for low-risk papillary thyroid microcarcinoma: more than 5-year follow-up for 84 tumors. Thyroid 2020;30:17451751

42. Chen L, Wu YH, Lee CH, Chen HA, Loh EW, Tam KW. Prophylactic central neck dissection for papillary thyroid carcinoma with clinically uninvolved central neck lymph nodes: a systematic review and meta-analysis. World J Surg 2018;42:2846-2857 